\title{
Adesão à farmacoterapia em hipertensos cadastrados na Estratégia Saúde da Família
}

\author{
Adherence to pharmacotherapy in hypertensive subjects followed by the Family Health \\ Strategy
}

Adhesion a la farmacoterapia a pacientes hipertensos inscritos en la Estrategia de Salud Familiar

Maria Nelly Sobreira de Carvalho Barreto ${ }^{1,3}$, Nathália Paula de Souza ${ }^{4}$, Silvia Pereira da Silva de Carvalho Melo $^{3}$, Heloisa de Melo Rodrigues ${ }^{5}$, Annick Fontbonne ${ }^{6}$, Eduarda Ângela Pessoa Cesse ${ }^{3}$.

\section{RESUMO}

Objetivo: Analisar os fatores associados à adesão à farmacoterapia em usuários hipertensos, assim como sua relação com o controle pressórico. Métodos: Trata-se de um estudo transversal com abordagem quantitativa, baseado nos dados do estudo RedeNut, conduzido na Estratégia Saúde da Família nos municípios polos das macrorregiões de saúde de Pernambuco, em 2015-16. Foram sorteados 404 usuários acima de 20 anos. O escore de adesão baseou-se num instrumento validado para este fim, o Brief Medication Questionnaire. Resultados: A amostra analisada foi de 339 hipertensos, sendo 68,4\%-mulheres. 48,8\% tinham a pressão controlada e 45,4\%, eram hipertensos e diabéticos. $60 \%$ dos hipertensos foram considerados aderentes ao tratamento farmacológico. Na análise multivariada, as variáveis associadas, positivamente, à adesão foram, a idade $(O R=1,028 ; p=0,021)$ e a renda familiar mensal $(O R=2,147 ; p=0,060)$, e negativamente, a politerapia antidiabética $(O R=0,270 ; p=0,010)$ e a dificuldade em tomar vários medicamentos $(O R=0,357 ; p=0,038)$. Conclusão: Os resultados demonstraram que os fatores socioeconômicos e demográficos, bem como os relacionados à farmacoterapia e algumas limitações dos usuários, influenciam na adesão ao tratamento farmacológico. Recomenda-se, assim, a adoção de estratégias para aprimorar a adesão.

Palavras-chave: Adesão à medicação, Atenção Primária à Saúde, Hipertensão.

\section{ABSTRACT}

Objective: To analyze the associated factors with adherence to pharmacotherapy in hypertension users, as well as its relationship with blood pressure control. Methods: This was a cross-sectional study, conducted with a quantitative approach and based on data collected from the RedeNut study, conducted in the Family Health Strategy of the cities heading the health regions of Pernambuco, in 2015-16. A total of 404 users over 20 years old were selected. The adherence score was based on a validated instrument, the Brief Medication Questionnaire. Results: The analyzed sample was 339 hypertensive subjects, $68,4 \%$ of women; $48.8 \%$ had their blood pressure controlled and $45.4 \%$ were also diabetic. Sixty percent were considered adherent to pharmacological treatment. In multivariate analysis, variables significantly associated with adherence were, positively, age $(O R=1.028 ; p=0.021)$ and monthly family income $(O R=2.147 ; p=0.060)$, and, negatively, polytherapy for diabetes $(O R=0.270 ; p=0.010)$ and difficulty in taking many medicines $(\mathrm{OR}=0.357 ; \mathrm{p}=0.038)$. Conclusion: The results showed that socioeconomic and demographic factors, as well as related to pharmacotherapy and some users' limitations, influenced adherence to pharmacological treatment. Thus, it is recommended to adopt strategies to improving adherence.

Keywords: Medication Adherence, Primary Health Care, Hypertension.

\footnotetext{
${ }^{1}$ Faculdade Pernambucana de Saúde, Recife - PE. `E-mail: nellyscbarreto@gmail.com

${ }^{2}$ Prefeitura Municipal de Recife, Recife - PE.

${ }^{3}$ Instituto Aggeu Magalhães (IAM), Fundação Oswaldo Cruz (FIOCRUZ), Recife - PE.

${ }^{4}$ Universidade Federal de Pernambuco (UFPE), Vitória de Santo Antão - PE.

${ }^{5}$ Instituto Federal de Educação, Ciência e Tecnologia de Pernambuco, Recife - PE.

${ }^{6}$ Centro de Pesquisa em Epidemiologia e Saúde das Populações (CESP), INSERM U1018, Villejuif - França.

O estudo integra a pesquisa "RedeNut: Avaliação da inserção do Componente Alimentação e Nutrição na rede de atenção aos hipertensos e diabéticos em Pernambuco", a qual foi financiada pela FACEPE (APQ0277-4.06-13) e pelo CNPq (Programa Ciência sem Fronteiras: 406640/2013-0; 301513/2014-7)
} 


\section{RESUMEN}

Objetivo: Analizar los factores asociados a la adhesión a la farmacoterapia en usuarios con hipertensión, y su relación con el control de la presión arterial. Métodos: Se trata de un estudio transversal con un enfoque cuantitativo, basado en datos del estudio RedeNut, realizado en la Estrategia de Salud Familiar en los municipios centrales de las macrorregiones sanitarias de Pernambuco, en 2015-16. Se seleccionaron 404 usuarios mayores de 20 años. La puntuación de la adherencia se basó en un instrumento validado para este fin, el Brief Medication Questionnaire. Resultados: La muestra analizada fue de 339 pacientes hipertensos, 68,4\% mujeres. 48,8\% tenían presión controlada y $45,4 \%$ eran hipertensos y diabéticos. $60 \%$ fueron clasificados como adherentes al tratamiento farmacológico. En el análisis multivariado, las variables significativamente asociadas, positivamente con la adherencia fueron la edad $(O R=1,028 ; p-0,021)$ y los ingresos familiares mensuales $(O R-2,147 ; p-0,060)$, y negativamente fueron, la politerapia antidiabética (OR=0,270; $p-0,010)$ y la dificultad para tomar varios medicamentos $(\mathrm{OR}=0,357 ; \mathrm{p}-0,038)$. Conclusión: Los resultados mostraron que factores socioeconómicos y demográficos, así como relacionados con la farmacoterapia y algunas limitaciones de los usuarios, influyen en la adherencia al tratamiento farmacológico. Por ello, se recomienda adoptar estrategias capaces de mejorar la adhesión,

Palabras clave: Adhesión al tratamiento, Atención Primaria en Salud, Hipertensión.

\section{INTRODUÇÃO}

A Organização Mundial de Saúde (WORLD HEALTH ORGANIZATION, 2018) estimou que 25\% da população mundial tem hipertensão arterial sistêmica (HAS). Malta DC, et al. (2016) com base nos dados da Pesquisa Nacional de Saúde, realizada em 2013, identificaram que 22,8\% da população brasileira tem pressão arterial aferida maior ou igual a $140 / 90 \mathrm{mmHg}$, sendo mais elevada em homens $(25,8 \%)$ do que em mulheres $(20,0 \%)$. A HAS, por sua condição multifatorial e associação frequente aos distúrbios metabólicos, causa alterações funcionais e/ou estruturais de órgãos-alvo, fato que se torna ainda mais grave quando ocorre concomitante ao diabetes mellitus (DM).

$O$ uso contínuo de anti-hipertensivos, aliado às mudanças no estilo de vida, é fundamental para o controle da hipertensão e representa meta prioritária para redução das complicações (MALACHIAS MVB, et al., 2017; SOCIEDADE BRASILEIRA DE DIABETES, 2019). Todavia, por ser uma doença silenciosa, algumas vezes o tratamento é negligenciado. Estudos nacionais e internacionais relatam as causas multifatoriais que podem afetar a adesão à farmacoterapia (MEINERS MMMA, et al., 2017; MZOUGHI K et al., 2018; RAJPURA J e NAYAK R, 2014; STOPA SR, et al., 2018).

De acordo com Reach G (2018) a adesão tem relação com o princípio da autonomia e pode ser intencional (processo ativo, relacionado à decisão do paciente) e não intencional (processo passivo, por exemplo, habilidades cognitivas, regime complexo, idade, dificuldades financeiras ou de acesso aos medicamentos). Desta forma, é imprescindível reforçar os cuidados primários que favoreçam o cumprimento das medidas farmacológicas e não farmacológicas. No SUS, estes cuidados são subsidiados pela Atenção Primária à Saúde (APS), que conta com o apoio dos profissionais da Estratégia Saúde da Família (ESF).

Assim, é indispensável identificar os fatores que influenciam a adesão à farmacoterapia a fim de que se possa estabelecer estratégias, em parceria com a equipe de saúde da família e o usuário, capazes de favorecer a continuidade do tratamento (BRASIL, 2011; GEWEHR DM, et al., 2018; MALACHIAS MVB, et al., 2017).

É provável que esses fatores sejam diferentes entre regiões do Brasil. Uma análise das desigualdades regionais realizada por Albuquerque MV, et al. (2017), no período de 2000 a 2016, apesar de apontar para avanços na redução da pobreza e das desigualdades nos anos 2000 , destacou efeito paradoxal sobre 0 território brasileiro, sendo que a distribuição dos municípios com muita pobreza permaneceu concentrada no Norte e Nordeste.

Essas desigualdades regionais influenciam no acesso, também desigual, aos medicamentos destinados aos hipertensos e diabéticos, como observado por Barreto MNSC, et al. (2015) e que pode impactar na adesão ao tratamento. Neste contexto, conduziu-se uma análise em usuários com hipertensão cadastrados na Estratégia Saúde da Família do Estado de Pernambuco, com o objetivo de identificar as variáveis relacionadas à adesão à farmacoterapia, assim como a influência destes aspectos na saúde dos hipertensos e, consequentemente, no tratamento farmacológico e controle pressórico. 


\section{MÉTODOS}

Trata-se de um estudo transversal, analítico com abordagem quantitativa, realizado com base nos dados do estudo epidemiológico "RedeNut: Avaliação da inserção do Componente Alimentação e Nutrição na rede de atenção aos hipertensos e diabéticos em Pernambuco", desenvolvido pelo Instituto Aggeu Magalhães, Fiocruz-Pernambuco.

Foi calculado um tamanho amostral mínimo de 300 pessoas para estimar com precisão de $6 \%$ a proporção de usuários bem informados sobre esses aspectos de seu tratamento, tendo em conta um efeito de conglomerado de 1,5. A coleta de dados ocorreu no período de agosto de 2015 a setembro de 2016 e foi realizada nos quatro polos de macrorregiões de saúde do Estado de Pernambuco: Recife, Caruaru, Serra Talhada e Petrolina.

A amostra foi constituída em duas etapas. Na primeira etapa, realizou-se um levantamento no Cadastro Nacional de Estabelecimentos de Saúde (CNES), em que se constatou haver 399 equipes atuando na ESF, em abril de 2015, nos quatro municípios alvo da pesquisa. Foram selecionadas, de forma aleatória, $25 \%$ destas equipes, proporcionalmente ao número de equipes de cada polo.

Assim, 61 equipes foram sorteadas em Recife, 16 em Caruaru, 05 em Serra Talhada e 19 em Petrolina, totalizando 101 equipes. O tamanho da amostra permitia a comparação entre a capital Recife e os municípios do interior, que compreendem as cidades de Caruaru, Serra Talhada e Petrolina).

$\mathrm{Na}$ segunda etapa, em cada equipe participante, selecionou-se, a partir da listagem do Agente Comunitário de Saúde (ACS), uma amostra aleatória de dois usuários hipertensos e dois usuários diabéticos acima de 20 anos, de ambos os sexos, para realização de entrevistas, totalizando 404 usuários sorteados. Para análise da adesão ao tratamento farmacológico foram selecionados os hipertensos, com ou sem diabetes, que estavam em farmacoterapia no momento da entrevista.

Foram excluídos da análise os usuários que se recusaram a participar $(n=9)$, os que eram apenas diabéticos ou não estavam em uso de anti-hipertensivos no momento da entrevista $(n=49)$ e, também, os questionários que apresentaram inconsistência $(n=7)$.

Entrevistadores, devidamente treinados, aplicaram formulários padronizados, construídos, testados e validados internamente antes da coleta de dados no campo. A adesão à farmacoterapia foi considerada a variável dependente deste estudo, sendo obtida por meio da aplicação de questionários, embasados na versão em português do teste Brief Medication Questionnaire (BMQ), um instrumento composto por perguntas organizadas em 11 escores e dividido em três domínios que identificam as barreiras para adesão quanto ao regime de tratamento prescrito (sete escores), as crenças (dois escores) e a recordação (dois escores) em relação ao tratamento medicamentoso na perspectiva do paciente (BEN AJ, et al., 2012).

O domínio regime avalia o comportamento do paciente em relação ao regime prescrito, considerando-se a dose diária, omissão ou redução da dose e interrupção do tratamento. O domínio crença avalia a crença dos pacientes na eficácia do tratamento e seus efeitos colaterais indesejáveis. O terceiro domínio, recordação, identifica problemas em relação à lembrança em tomar o medicamento, a partir do questionamento sobre o quanto é difícil lembrar de tomar os fármacos e revisa o regime de tomada das medicações.

A adesão ao tratamento farmacológico foi categorizada, a partir do escore calculado, em: aderente (não respondeu afirmativamente nenhuma pergunta), provavelmente aderente (respondeu afirmativamente apenas uma pergunta), provavelmente pouco aderente (respondeu afirmativamente duas perguntas), e pouco aderente (respondeu afirmativamente três ou mais perguntas).

Estes resultados foram agrupados em duas categorias, 'alta adesão' que agrupou os aderentes e provavelmente aderentes, ou seja, os que tiveram de zero a um ponto nos três domínios, e 'baixa adesão' que agrupou os provavelmente pouco aderentes e os pouco aderentes, ou seja os que apresentaram pontuação de dois pontos ou mais. 
A pressão arterial (PA) foi aferida duas vezes, durante a entrevista, utilizando tensiômetros digitais de pulso. Quando a diferença entre a primeira e segunda medida, tanto para a pressão arterial sistólica (PAS) como para a pressão arterial diastólica (PAD), era superior à $4 \mathrm{mmHg}$, realizava-se uma terceira aferição, tirando uma média das PAs aferidas. A classificação do controle da PA seguiu as orientações da VII Diretriz Brasileira de Hipertensão (MALACHIAS MVB, et al., 2017).

A partir dos valores obtidos, os participantes não diabéticos foram divididos em dois grupos: o primeiro grupo, classificado com PA controlada foi constituído por usuários que apresentaram PAS $<140 \mathrm{mmHg}$ e PAD $<90 \mathrm{mmHg}$ e o segundo grupo foi caracterizado com PA não controlada, tendo em vista que os indivíduos apresentavam PAS $\geq 140 \mathrm{mmHg}$ ou $\mathrm{PAD} \geq 90 \mathrm{mmHg}$.

A partir da construção dos escores de adesão foi possível realizar uma análise comparativa da alta ou baixa adesão com os fatores socioeconômicos e demográficos, os aspectos clínicos e farmacológicos (esquemas de tratamento com anti-hipertensivos e antidiabéticos), as dificuldades relatadas pelos hipertensos na condução do tratamento (classificadas em não tem, tem pouca ou tem muita dificuldade), assim como as percepções dos entrevistadores quanto às limitações apresentadas pelos usuários e sua influência na adesão à farmacoterapia. Procurou-se, ainda, estabelecer uma correlação entre os domínios de BMQ com o controle pressórico.

Os dados coletados foram tabulados utilizando o programa EpiData 3.1 e compilados em tabelas por meio do software estatístico Statistical Package for the Social Sciences (SPSS) versão 19, para armazenamento e análise dos dados. As variáveis qualitativas/categóricas foram apresentadas por meio de suas frequências absoluta e relativa e as variáveis quantitativas por meio de média e desvio-padrão.

$\mathrm{Na}$ análise bivariada, a finalidade foi de verificar a associação entre a adesão ao tratamento farmacológico (variável dependente) e algumas das variáveis independentes do estudo que englobaram as variáveis socioeconômicas e demográficas, os aspectos clínicos e farmacológicos e as dificuldades encontradas pelos usuários na condução do seu tratamento farmacológico.

Para a análise estatística bivariada, utilizou-se o teste Qui-Quadrado ou teste exato de Fisher (quando necessário), no caso das variáveis qualitativas e o teste t de Student para as quantitativas. Além disso, foi de interesse investigar a associação entre os domínios do BMQ e a variável de adesão com o controle da pressão arterial, utilizando para tal o teste Qui-quadrado ou teste exato de Fisher, quando necessário. Todas as conclusões foram tomadas ao nível de $5 \%$ de significância (valor de $p<0,05$ ).

Para a análise multivariada, variáveis com associação estatisticamente significativa a $20 \%$ (valor de $p<$ $0,20)$ na análise bivariada entraram no modelo de regressão logística, com estatística de Wald, com o objetivo de determinar a contribuição de cada covariável para adesão ao tratamento.

O método de seleção das variáveis foi o backward, mantidas no modelo final as variáveis que apresentaram um nível de significância inferior a 10\%. Dessa forma, foi calculada a razão de chances ou odds ratio (OR) de ocorrência do evento (adesão ao tratamento) e respectivo intervalo de confiança (IC 95\%).

O estudo RedeNut foi aprovado pelo Comitê de Ética em Pesquisa da Fiocruz-Pernambuco e pelo Comitê Nacional de Ética em Pesquisa (CONEP/Conselho Nacional de Saúde/Ministério da Saúde) conforme CAAE: 21989413.0.0000.5190. Os usuários entrevistados foram convidados a assinar Termo de Consentimento Livre e Esclarecido (TCLE), e foram informados sobre a justificativa, os objetivos e os procedimentos utilizados na pesquisa.

\section{RESULTADOS}

Os resultados a seguir foram apresentados da seguinte forma: primeiro uma descrição com o intuito de conhecer as características dos entrevistados, seus aspectos clínicos, farmacológicos e as dificuldades encontradas para tomar os medicamentos, para posteriormente discutir as análises bivariadas, e multivariadas, tendo a adesão e o controle pressórico como variáveis dependentes. 
Dos 339 usuários selecionados, mais de $60 \%$ estavam vinculados às equipes da ESF de Recife. De acordo com as características da amostra total, a idade média dos entrevistados foi de $64,0 \pm 13,1$ anos, com predomínio das mulheres $(68,4 \%)$.

A média de anos de estudo formal foi de $6,3 \pm 3,6$, para os que declararam saber ler e escrever $(67,3 \%)$. a renda familiar mensal para a maioria $(40,7 \%)$, foi de até um salário mínimo houve predominância de $(61,7 \%)$ aposentados ou pensionistas e $67,8 \%$ referiram ser chefe de família (Tabela 1).

Tabela 1 - Análise bivariada entre as variáveis socioeconômicas, demográficas e a adesão ao tratamento farmacológico em hipertensos cadastrados na Estratégia de Saúde da Família no Estado de Pernambuco.

\begin{tabular}{|c|c|c|c|c|c|c|c|}
\hline \multirow{2}{*}{ Variáveis } & \multicolumn{2}{|c|}{ Amostra } & \multicolumn{2}{|c|}{ Baixa adesão } & \multicolumn{2}{|c|}{ Alta adesão } & \multirow{2}{*}{ Valor de $\mathrm{p}^{*}$} \\
\hline & $\mathrm{n}$ & $\%$ & $\mathrm{n}$ & $\%$ & $\mathrm{n}$ & $\%$ & \\
\hline \multicolumn{8}{|l|}{ Local de residência } \\
\hline Recife & 210 & 61,9 & 86 & 41,0 & 124 & 59,0 & \multirow{2}{*}{0,689} \\
\hline Interior & 129 & 38,1 & 50 & 38,8 & 79 & 61,2 & \\
\hline $\begin{array}{c}\text { Idade, em } \\
\text { anos }(m \pm D P)^{\star *}\end{array}$ & \multicolumn{2}{|c|}{$64,0 \pm 13,1$} & \multicolumn{2}{|c|}{$61,4 \pm 14,1$} & \multicolumn{2}{|c|}{$65,8 \pm 12,1$} & 0,003 \\
\hline \multicolumn{8}{|l|}{ Sexo } \\
\hline Masculino & 107 & 31,6 & 45 & 42,1 & 62 & 57,9 & \multirow{2}{*}{0,621} \\
\hline Feminino & 232 & 68,4 & 91 & 39,2 & 141 & 60,8 & \\
\hline \multicolumn{8}{|l|}{ Sabe ler e escrever } \\
\hline Não & 111 & 32,7 & 35 & 31,5 & 76 & 68,5 & \multirow{2}{*}{0,024} \\
\hline Sim & 228 & 67,3 & 101 & 44,3 & 127 & 55,7 & \\
\hline $\begin{array}{l}\text { Anos de estudo formal } \\
(m \pm D P)^{\star *}\end{array}$ & \multicolumn{2}{|c|}{$6,3 \pm 3,6$} & \multicolumn{2}{|c|}{$5,9 \pm 3,5$} & \multicolumn{2}{|c|}{$6,6 \pm 3,7$} & 0,165 \\
\hline \multicolumn{8}{|l|}{$\begin{array}{l}\text { Renda familiar mensal, em } \\
\text { salários mínimos (SM) }\end{array}$} \\
\hline Até $1 \mathrm{SM}$ & 138 & 40,7 & 60 & 43,5 & 78 & 56,5 & \multirow{3}{*}{0,053} \\
\hline Mais de 1 e até $2 \mathrm{SM}$ & 126 & 37,2 & 55 & 43,7 & 71 & 56,3 & \\
\hline Mais de 2 SM & 75 & 22,1 & 21 & 28,0 & 54 & 72,0 & \\
\hline \multicolumn{8}{|l|}{ Situação profissional } \\
\hline $\begin{array}{l}\text { Empregado (com ou sem } \\
\text { carteira) }\end{array}$ & 16 & 4,7 & 7 & 43,8 & 9 & 56,2 & \multirow{6}{*}{0,044} \\
\hline Autônomo / comerciante & 26 & 7,7 & 11 & 42,3 & 15 & 57,7 & \\
\hline Desempregado & 17 & 5,0 & 9 & 52,9 & 8 & 47,1 & \\
\hline Do lar & 51 & 15,0 & 25 & 49,0 & 26 & 51,0 & \\
\hline $\begin{array}{l}\text { Sem ocupação, com renda } \\
\text { (aposentado ou pensionista) }\end{array}$ & 209 & 61,7 & 71 & 34,0 & 138 & 66,0 & \\
\hline Outras ocupações & 20 & 5,9 & 13 & 65,0 & 7 & 35,0 & \\
\hline \multicolumn{8}{|l|}{ Chefe de família } \\
\hline Não & 109 & 32,2 & 47 & 43,1 & 62 & 56,9 & \multirow{2}{*}{0,438} \\
\hline Sim & 230 & 67,8 & 89 & 38,7 & 141 & 61,3 & \\
\hline
\end{tabular}

Legenda: *Teste Qui-quadrado (ou teste exato de Fisher) para variáveis qualitativas / Teste t de Student para variáveis quantitativas. ${ }^{* *} \mathrm{~m} \pm \mathrm{DP}$ : media \pm Desvio- Padrão

Fonte: Barreto MNSC, et al., 2021.

Em relação aos aspectos clínicos um pouco mais de $50 \%$ eram apenas hipertensos e apresentaram o

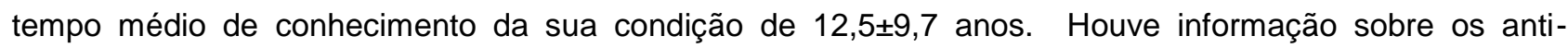
hipertensivos em uso por 334 hipertensos e a maioria $(64,4 \%)$ estavam em politerapia com dois ou mais medicamentos anti-hipertensivos. Dos 154 hipertensos que referiram ser também diabéticos, 133 estavam em uso de antidiabéticos, sendo a maioria em monoterapia (Tabela 2). 
Tabela 2 - Análise bivariada entre os aspectos clínicos e farmacológicos e a adesão ao tratamento farmacológico em hipertensos cadastrados na Estratégia de Saúde da Família no Estado de Pernambuco.

\begin{tabular}{|c|c|c|c|c|c|c|c|c|}
\hline \multirow{2}{*}{\multicolumn{2}{|c|}{ Variáveis }} & \multicolumn{2}{|c|}{ Amostra } & \multicolumn{2}{|c|}{ Baixa adesão } & \multicolumn{2}{|c|}{ Alta adesão } & \multirow{2}{*}{$\begin{array}{l}\text { Valor } \\
\text { de } p^{*}\end{array}$} \\
\hline & & $\mathrm{n}$ & $\%$ & $\mathrm{n}$ & $\%$ & $n$ & $\%$ & \\
\hline \multicolumn{2}{|c|}{$\begin{array}{l}\text { Há quanto tempo sabe que tem } \\
\text { hipertensão }(m \pm D P)^{\star \star}\end{array}$} & \multicolumn{2}{|c|}{$12,5 \pm 9,7$} & \multicolumn{2}{|c|}{$12,4 \pm 9,5$} & \multicolumn{2}{|c|}{$12,9 \pm 9,9$} & 0,683 \\
\hline Tem diabetes: & $\begin{array}{l}\text { Sim } \\
\text { Não }\end{array}$ & $\begin{array}{l}154 \\
185 \\
\end{array}$ & $\begin{array}{l}45,4 \\
54,6\end{array}$ & $\begin{array}{l}71 \\
65\end{array}$ & $\begin{array}{l}46,1 \\
35,1\end{array}$ & $\begin{array}{c}83 \\
120 \\
\end{array}$ & $\begin{array}{l}53,9 \\
64,9\end{array}$ & 0,040 \\
\hline \multicolumn{2}{|c|}{$\begin{array}{l}\text { Há quanto tempo sabe que tem } \\
\text { diabetes }(m \pm D P)^{\star \star}\end{array}$} & \multicolumn{2}{|c|}{$10,2 \pm 8,6$} & \multicolumn{2}{|c|}{$9,7 \pm 8,7$} & \multicolumn{2}{|c|}{$10,6 \pm 8,5$} & 0,532 \\
\hline \multicolumn{9}{|c|}{ № de anti-hipertensivos } \\
\hline \multicolumn{2}{|c|}{ Monoterapia } & $\begin{array}{l}119 \\
215\end{array}$ & $\begin{array}{l}35,6 \\
64,4\end{array}$ & $\begin{array}{l}46 \\
88\end{array}$ & $\begin{array}{l}38,7 \\
40,9\end{array}$ & $\begin{array}{c}73 \\
127 \\
\end{array}$ & $\begin{array}{l}61,3 \\
59,1\end{array}$ & 0,685 \\
\hline \multicolumn{9}{|c|}{ № de antidiabéticos } \\
\hline \multicolumn{2}{|c|}{$\begin{array}{c}\text { Nenhum } \\
\text { Monoterapia } \\
\text { Politerapia }\end{array}$} & $\begin{array}{l}206 \\
94 \\
39\end{array}$ & $\begin{array}{l}60,8 \\
27,7 \\
11,5\end{array}$ & $\begin{array}{l}73 \\
38 \\
25\end{array}$ & $\begin{array}{l}35,4 \\
40,4 \\
64,1\end{array}$ & $\begin{array}{c}133 \\
56 \\
14\end{array}$ & $\begin{array}{l}64,6 \\
59,6 \\
35,9\end{array}$ & 0,004 \\
\hline
\end{tabular}

Legenda: *Teste Qui-quadrado (ou teste exato de Fisher) para variáveis qualitativas / Teste t de Student para variáveis quantitativas ${ }^{\star *} \mathrm{~m} \pm \mathrm{DP}:$ media \pm Desvio- Padrão.

Fonte: Barreto MNSC, et al., 2021.

Menos da metade $(165 ; 48,8 \%)$ dos entrevistados estava com a PAS e PAD controlada. De acordo com o critério de adesão $\mathrm{BMQ}, 60 \%$ dos hipertensos foram classificados na categoria dicotomizada de 'alta adesão', porém considerados como provavelmente aderentes em sua maioria (42\%). Entre os $40 \%$ classificados com uma 'baixa adesão', $31,1 \%$ se enquadraram no critério de provavelmente pouco aderentes. No tocante aos domínios que compõem o BMQ, constatou-se uma maior adesão dos usuários no domínio crença $(81,1 \%)$, seguida do domínio regime $(61,5 \%)$ e recordação $(26,6 \%)$ (Tabela 3).

Tabela 3 - Associação entre os domínios do Brief Medication Questionnaire (BMQ) e o controle pressórico* em hipertensos cadastrados na Estratégia de Saúde da Família no Estado de Pernambuco.

\begin{tabular}{|c|c|c|c|c|c|c|c|}
\hline \multirow[t]{2}{*}{ Variáveis } & \multicolumn{2}{|c|}{ Amostra } & \multicolumn{2}{|c|}{$\begin{array}{l}\text { HAS controlada } \\
(n=165)\end{array}$} & \multicolumn{2}{|c|}{$\begin{array}{l}\text { HAS não controlada } \\
(n=173)\end{array}$} & \multirow[t]{2}{*}{$\begin{array}{l}\text { Valor } \\
\text { de } p^{\star \star}\end{array}$} \\
\hline & $\mathrm{n}$ & $\%$ & $\mathrm{n}$ & $\%$ & $\mathrm{n}$ & $\%$ & \\
\hline \multicolumn{8}{|l|}{ Categorias dicotomizadas } \\
\hline Baixa adesão & 135 & 40,0 & 67 & 40,6 & 68 & 39,3 & \multirow{2}{*}{0,807} \\
\hline Alta adesão & 203 & 60,0 & 98 & 59,4 & 105 & 60,7 & \\
\hline \multicolumn{8}{|l|}{ Categorias de adesão BMQ } \\
\hline Aderente & 61 & 18,0 & 32 & 19,4 & 29 & 16,8 & \multirow{4}{*}{0,880} \\
\hline Provavelmente aderente & 142 & 42,0 & 66 & 40,0 & 76 & 43,9 & \\
\hline Provavelmente pouco aderente & 105 & 31,1 & 52 & 31,5 & 53 & 30,6 & \\
\hline Pouco aderente & 30 & 8,9 & 15 & 9,1 & 15 & 8,7 & \\
\hline \multicolumn{8}{|l|}{ Domínio Regime } \\
\hline Alta adesão & 208 & 61,5 & 102 & 61,8 & 106 & 61,3 & \multirow{2}{*}{0,918} \\
\hline Baixa adesão & 130 & 38,5 & 63 & 38,2 & 67 & 38,7 & \\
\hline \multicolumn{8}{|l|}{ Domínio Crenças } \\
\hline Alta adesão & 274 & 81,1 & 135 & 81,8 & 139 & 80,3 & \multirow{2}{*}{0,730} \\
\hline Baixa adesão & 64 & 18,9 & 30 & 18,2 & 34 & 19,7 & \\
\hline \multicolumn{8}{|l|}{ Domínio Recordação } \\
\hline Alta adesão & 90 & 26,6 & 43 & 26,1 & 47 & 27,2 & \multirow{2}{*}{0,818} \\
\hline Baixa adesão & 248 & 73,4 & 122 & 73,9 & 126 & 72,8 & \\
\hline
\end{tabular}

Legenda: HAS: Hipertensão Arterial Sistêmica

* Não foi possível classificar o controle pressórico de um hipertenso

** Teste Qui-quadrado (ou teste exato de Fisher)

Fonte: Barreto MNSC, et al., 2021. 
As principais dificuldades relacionadas à farmacoterapia, citadas pelos entrevistados em ordem decrescente, foram a leitura das informações contidas nas embalagens $(72,1 \%)$, o quantitativo de comprimidos tomados diariamente $(33,9 \%$,) e o manuseio da embalagem $(13,4 \%)$ (Tabela 4).

Outro aspecto considerado foram as limitações dos hipertensos percebidas pelo entrevistador. A mais citada delas foi o analfabetismo (26,3\%), seguida de problemas emocionais, como sintomas depressivos $(20,6 \%)$ e diminuição da acuidade visual ou auditiva $(20,0 \%)$. Os entrevistadores não identificaram nenhuma limitação em apenas 5,6\% dos entrevistados (informação não apresentada em tabela).

Tabela 4 - Análise bivariada entre as dificuldades para tomada de medicamentos e a adesão ao tratamento farmacológico em hipertensos cadastrados na Estratégia de Saúde da Família no Estado de Pernambuco.

\begin{tabular}{|c|c|c|c|c|c|c|c|}
\hline \multirow[t]{2}{*}{ Variáveis } & \multicolumn{2}{|c|}{ Amostra } & \multicolumn{2}{|c|}{$\begin{array}{c}\text { Baixa } \\
\text { adesão }\end{array}$} & \multicolumn{2}{|c|}{$\begin{array}{c}\text { Alta } \\
\text { adesão }\end{array}$} & \multirow[t]{2}{*}{ Valor de $p^{\star}$} \\
\hline & $\mathrm{n}$ & $\%$ & $\mathrm{n}$ & $\%$ & $\mathrm{n}$ & $\%$ & \\
\hline \multicolumn{8}{|c|}{ Dificuldade de abrir ou fechar a embalagem } \\
\hline Não tem dificuldade & 284 & 86,6 & 109 & 38,4 & 175 & 61,6 & \multirow{3}{*}{0,338} \\
\hline Pouca dificuldade & 26 & 7,9 & 11 & 42,3 & 15 & 57,7 & \\
\hline Muita dificuldade & 18 & 5,5 & 10 & 55,6 & 8 & 44,4 & \\
\hline \multicolumn{8}{|c|}{ Dificuldade de ler o que está escrito na embalagem } \\
\hline Não tem dificuldade & 81 & 27,8 & 31 & 38,3 & 50 & 61,7 & \multirow{3}{*}{0,170} \\
\hline Pouca dificuldade & 83 & 28,5 & 29 & 34,9 & 54 & 65,1 & \\
\hline Muita dificuldade & 127 & 43,6 & 60 & 47,2 & 67 & 52,8 & \\
\hline \multicolumn{8}{|c|}{ Dificuldade de tomar tantos comprimidos ao mesmo tempo } \\
\hline Não tem dificuldade & 218 & 66,1 & 61 & 28,0 & 157 & 72,0 & \multirow{3}{*}{0,001} \\
\hline Pouca dificuldade & 73 & 22,1 & 49 & 67,1 & 24 & 32,9 & \\
\hline Muita dificuldade & 39 & 11,8 & 20 & 51,3 & 19 & 48,7 & \\
\hline
\end{tabular}

Legenda: *Teste Qui-quadrado (ou teste exato de Fisher).

Fonte: Barreto MNSC, et al., 2021.

$\mathrm{Na}$ análise bivariada, os hipertensos com maior idade $(p=0,003)$, não alfabetizados $(p=0,024)$, com renda superior a dois salários mínimos ( $p=0,053)$ e que mesmo sem ocupação, possuíam alguma fonte de renda (aposentados/pensionistas e correlatos) ( $(=0,044)$, apresentaram associação significativa com alta adesão ao tratamento farmacológico. Quanto à situação clínica, observou-se maior adesão entre os hipertensos que não tinham diabetes $(p=0,040)$, não tomavam nenhum antidiabético $(p=0,004)$ não tinham dificuldade em tomar vários medicamentos $(\mathrm{p}=0,001)$.

Quanto às limitações referidas pelos entrevistadores, enquanto 0 analfabetismo influenciou positivamente $(p=0,026)$ na alta adesão, os problemas emocionais tiveram uma maior relação com a baixa adesão ao tratamento farmacológico $(p=0,003)$. Não se evidenciou relação entre $\circ$ grau de adesão e 0 controle da pressão arterial. $\mathrm{Na}$ análise multivariada, os fatores sociodemográficos e econômicos que permaneceram no modelo final foram a idade $(O R=1,028 ; p=0,021)$ e a renda superior a dois salários mínimos $(O R=2,147 ; p=0,060)$, associadas positivamente à adesão.

Entre os aspectos clínicos permaneceram no modelo as variáveis referentes ao número de medicamentos antidiabéticos $(\mathrm{OR}=0,27 ; \mathrm{p}=0,010)$ e dificuldade em tomar muitos comprimidos ao mesmo tempo $(\mathrm{OR}=0,357 ; \mathrm{p}=0,038)$, relacionados à menor grau de adesão.

\section{DISCUSSÃO}

Neste estudo conduzido numa amostra aleatória de hipertensos acompanhados pela Estratégia Saúde da Família nos quatro polos de macrorregiões de saúde de Pernambuco, mais da metade $(60 \%)$ dos participantes se enquadraram no critério de adesão ao tratamento farmacológico. 
Vale ressaltar, todavia, que a grande maioria destes estava na classe de 'provável adesão'. Esses resultados condizem com outros estudos realizados no Brasil, que também investigaram a adesão à farmacoterapia (BARRETO MS, et al., 2016; GEWEHR DM, et al., 2018; TAVARES DMS, et al., 2016). Considerando os três domínios do $\mathrm{BMQ}$, o que mais influenciou para adesão foi o domínio crença, seguido do domínio regime e recordação. Gewehr DM, et al. (2018), que utilizaram o mesmo instrumento, encontraram resultados semelhantes quanto à adesão nos três domínios.

No que diz respeito à investigação dos fatores relacionados à adesão ao tratamento farmacológico, impactaram positivamente na adesão à farmacoterapia os seguintes fatores: ter idade mais avançada, ter renda familiar acima de dois salários mínimos; e negativamente, um maior número de medicamentos antidiabéticos e a dificuldade em tomar vários comprimidos diariamente.

$\mathrm{O}$ aumento da idade esteve associado à adesão ao tratamento farmacológico, corroborando os achados de Lefort M, et al. (2018), Boratas S e Kilic HF (2018) que também verificaram uma maior adesão com o aumento da idade, atribuindo-se ao fato de que isto influencia numa maior preocupação com o estado de saúde. Todavia, em outros estudos no Brasil houve uma relação inversa entre a idade e a adesão ao tratamento farmacológico (BARRETO MS, et al., 2016; GEWEHR DM, et al., 2018).

A dificuldade da adesão com o aumento da idade foi justificada por autores que destacaram as diversas vulnerabilidades que surgem nos idosos, entre elas os problemas visuais ou auditivos e outras dificuldades físicas, além do declínio das capacidades mentais e cognitivas, o que contribui para o esquecimento ou dificulta a compreensão quanto ao uso dos medicamentos e, consequentemente, afeta o controle pressórico (TAVARES DMS, et al., 2016; GEWEHR DM, et al.2018).

Quanto à influência do nível socioeconômico, os resultados da análise bivariada apontaram para um grande percentual de pessoas sem ocupação, com renda, ou seja, aposentados, pensionistas etc., além dos não alfabetizados, que se enquadraram nos critérios de alta adesão à farmacoterapia. Esses fatores não permaneceram no modelo multivariado, quando ajustado pela idade, à qual estão ligados. Todavia, a renda acima de dois salários mínimos permaneceu como fator socioeconômico independente e positivamente associado à adesão, apesar da significância limítrofe $(p=0,060)$.

Nesse contexto, Santos IM, et al. (2020), analisaram o conhecimento e atitude dos usuários em relação a DM e constataram a influência da baixa renda, maior idade e baixo grau de escolaridade para o enfrentamento da doença, inferindo-se que estes fatores podem impactar negativamente na adesão ao tratamento.

Vale notar também, que quando mencionado enquanto limitação percebida pelos entrevistadores, o analfabetismo confirmou ter uma relação positiva com a alta adesão, enquanto os problemas emocionais como depressão e outras barreiras psicológicas foram relacionados com baixa adesão.

Outros estudos destacaram os sintomas depressivos, entre os fatores que dificultaram a adesão à farmacoterapia, (BARRETO MS, et al., 2016; LOR M, et al., 2019; MEINERS MMMA, et al., 2017). Estes achados alertam para a necessidade de um maior suporte da equipe de saúde e dos familiares visando identificar os problemas físicos, cognitivos e emocionais que impactam na qualidade de vida do idoso, refletindo na adesão à farmacoterapia. Os hipertensos com diabetes apresentaram menor adesão ao tratamento farmacológico, principalmente quando em politerapia com antidiabéticos, fator acrescentado à dificuldade referida pelos entrevistados, sobre ter que tomar tantos comprimidos ao mesmo tempo, que também estava associada à baixa adesão.

Estudos nacionais e internacionais mostram que os esquemas com múltiplos fármacos dificultam a adesão à farmacoterapia (BARRETO MS, et al., 2016; GEWEHR DM, et al., 2018; LEFORT M., et al.,2018). Outros estudos realizados constataram que um maior tempo de diagnóstico implica menor adesão e atribuíram causas, como o caráter crônico da doença, o uso de associação de fármacos e a presença de outras comorbidades (BARRETO MS, et al., 2016; GEWEHR DM, et al., 2018; TAVARES DMS, et al., 2016). 
De fato, as Diretrizes brasileiras de hipertensão (MALACHIAS MVB, et al., 2017) e de diabetes (SOCIEDADE BRASILEIRA DE DIABETES, 2019), enfatizam a tendência para inclusão de antihipertensivos e/ou antidiabéticos de classes diferentes à medida que a doença evolui. Tavares DMS, et al. (2016) identificaram, através de uma pesquisa com 1.029 idosos residentes em Uberaba/MG, que a maioria dos que não aderiram ao tratamento tinham mais comorbidades associadas.

Vale ressaltar que a adesão muito baixa no domínio recordação, identificada no presente estudo, pode se justificar pela idade média dos entrevistados, assim como pela quantidade de comprimidos que se toma em um dia. Os resultados encontrados não permitiram estabelecer uma associação entre a adesão e o controle pressórico aferido no momento da entrevista. Diversos estudos internacionais e nacionais, referiram dificuldade em controlar a pressão arterial entre os hipertensos que não aderiram ao tratamento farmacológico (BARRETO MS, et al., 2016; DURAND H, et al., 2017; GEWEHR DM, et al., 2018; RAJPURA J e NAYAK R, 2014;).

A divergência com a presente análise pode ser devido a algumas limitações do estudo base como, desenho transversal, que não permite estabelecer relação de causa e efeito entre as variáveis associadas, e verificação da PA, no momento da entrevista, visto que os níveis tensionais sofrem influências momentâneas do ambiente, da alimentação e do estado emocional do indivíduo; todavia, para minimizar esse problema, a PA foi aferida mais de uma vez.

Entre as forças do estudo destacam-se a seleção aleatória da amostra, para a população vinculada à ESF em quatro polos regionais de Pernambuco, e a utilização de um instrumento validado no Brasil, o BMQ, aplicado em diversos estudos, permitindo a comparação entre eles. No entanto, sabe-se da dificuldade para mensurar adesão, motivo pelo qual vários estudos que abordaram adesão ao tratamento utilizaram mais de um instrumento.

Outro problema refere-se à coleta de dados, pois estes foram obtidos das declarações feitas pelos sujeitos da pesquisa que, devido ao nível de escolaridade e outras fragilidades pessoais, puderam ter dificuldades em responder a algumas perguntas do extenso formulário. Para minimizar esses vieses foi contratada uma equipe experiente de entrevistadores e o formulário foi pré-testado. As limitações do desenho transversal e da aferição da PA no momento da entrevista já foram mencionadas anteriormente.

\section{CONCLUSÃO}

Os resultados demonstraram que os fatores socioeconômicos e demográficos, assim como relacionados à farmacoterapia e aspectos psicológicos, influenciam na adesão ao tratamento farmacológico. A taxa de baixa adesão encontrada, apesar de condizente com outros estudos no Brasil e além do Brasil, alerta para a persistente necessidade de intervenções conjuntas entre a equipe de saúde e familiares no incentivo ao uso contínuo desses medicamentos. Portanto, só fornecer o medicamento não é suficiente, torna-se necessário identificar as dificuldades encontradas pelo usuário com relação ao seu tratamento e contribuir para minimizar os problemas, de forma a otimizar os investimentos na saúde e prevenir a ocorrência de complicações em uma população com vulnerabilidades socioeconômicas.

\section{AGRADECIMENTOS E FINANCIAMENTO}

Os autores agradecem à FACEPE- Fundação de Amparo a Ciência e Tecnologia do Estado de Pernambuco (APQ-0277-4.06-13) e ao CNPq (Programa Ciência sem Fronteiras: 406640/2013-0; 301513/2014-7) pelo financiamento da pesquisa RedeNut.

\section{REFERÊNCIAS}

1. ALBUQUERQUE MV, et al. Desigualdades regionais na saúde: mudanças observadas no Brasil de 2000 a 2016. Cien Saude Colet 2017; 22(4): 1055-64.

2. BARRETO MNSC, et al. Análise do acesso ao tratamento medicamentoso para hipertensão e diabetes na Estratégia Saúde da Família no Estado de Pernambuco, Brasil. Rev Bras Epidemiol 2015; 18: 413-24. 
3. BARRETO MS, et al. Fatores associados ao inadequado controle pressórico em pacientes da atenção primária. Esc Anna Nery 2016; 20(1): 114-20.

4. BEN AJ, et al. Teste de Morisky-Green e Brief Medication Questionnaire para avaliar adesão a medicamentos. Rev Saude Publica 2012; 46(2): 279-89.

5. BORATAS S, KILIC HF. Evaluation of medication adherence in hypertensive patients and influential factors. Pak J Med Sci 2018; 34(4): 959-63.

6. BRASIL. Ministério da Saúde. Secretaria de Vigilância à Saúde. Plano de ações estratégicas para o enfrentamento das Doenças Crônicas Não Transmissíveis no Brasil, 2011-2022. Brasília: Ministério da Saúde; 2011.

7. DURAND $\mathrm{H}$, et al. Medication adherence among patients with apparent treatment-resistent hypertension: systematic review and meta-analysis. J Hypertens 2017; 35(12): 2346-57.

8. GEWEHR DM, et al. Adesão ao tratamento farmacológico da hipertensão arterial na Atenção Primária à Saúde. Rev Saude Debate 2018; 42: 179-90.

9. LEFORT M, et al. Sex differences in adherence to antihypertensive treatment in patients aged above 55: The French League Against Hypertension Survey (FLAHS). J Clin Hypertens 2018; 20: 1496-1503.

10. LOR M, et al. Association between health literacy and medication adherence among Hispanics with hypertension. $J$ Racial Ethn Health Disparities 2019; 6(3): 517-24.

11. MALACHIAS MVB, et al. 7a Diretriz Brasileira de Hipertensão, 2017;24(1): 1-90.

12. MALTA DC, et al. Prevalence of high blood pressure measured in the Brazilian population, National Health Survey, 2013. São Paulo Med J 2016; 134: 163-70.

13. MEINERS MMMA, et al. Acesso e adesão a medicamentos entre pessoas com diabetes no Brasil: evidências da PNAUM. Rev Bras Epidemiol 2017; 20: 445-59.

14. MZOUGHI K, et al. Etude des facteurs de mauvaise observance médicamenteuse chez les hypertendus. Tunis Med 2018; 96: 385-90.

15. RAJPURA J, NAYAK R. Medication adherence in a sample of elderly suffering from hypertension: evaluating the influence of illness perceptions, treatment beliefs, and illness burden. J Manage Care Pharm 2014; 20: 58-65.

16. REACH G. Les défis de l'observance dans les maladies chroniques. Médecine des maladies Métaboliques 2018; 12: 511-15.

17. SANTOS IM, et al. Conhecimento e atitudes de usuários com Diabetes Mellitus em uma unidade de ambulatório especializada. Revista Eletrônica Acervo Saúde / Electronic Journal Collection Health. 2020.

18. SOCIEDADE BRASILEIRA DE DIABETES. Diretrizes da Sociedade Brasileira de Diabetes 2019-2020. São Paulo: Editora Clannad; 2019.

19. STOPA SR, et al. Prevalência da hipertensão arterial, do diabetes mellitus e da adesão às medidas comportamentais no Município de São Paulo, Brasil, 2003-2015. Cad Saude Publica 2018; 34(10): e00198717.

20. TAVARES DMS, et al. Qualidade de vida e adesão ao tratamento farmacológico entre idosos hipertensos. Rev Bras Enferm 2016; 69(1): 134-41.

21. WORLD HEALTH ORGANIZATION. Noncommunicable diseases country profiles 2018. Geneva: World Health Organization; 2018. 